\title{
MODELO DE EVALUACIÓN DE SITUACIONES TÁCTICAS EN EL FÚTBOL.
}

\author{
Caicedo. P, Sergio Andrés ${ }^{1}$, Vera. R, José Luis ${ }^{2}$, Ortega. P, Arles Javier ${ }^{3}$
}

\begin{abstract}
Sergio Andrés Caicedo: ${ }^{1}$ Magister en Ciencias de la Actividad Física y el deporte. Docente programa Educación física recreación y deportes. Universidad de Pamplona sergio.caicedo@unipamplona.edu.co

José Luis Vera Rivera 2: Doctor en ciencias de la cultura física y el deporte, profesor Tiempo completo Universidad del Valle Cali Valle del Cauca Colombia área educación física y deporte, grupo de investigación GRINDER. Vera.jose@correounivalle.edu.co.

Arles Javier Ortega Parra 3: Doctor en ciencias de la Cultura física y el deporte, profesor tiempo completo Universidad de Pamplona Norte de Santander Colombia, grupo de investigación actividad física y deporte. arlesjavierortegap@hotmail.com.
\end{abstract}

\section{RESUMEN:}

En el fútbol mundial actual se colocan en los primeros lugares, aquellos equipos que además de tener figuras relevantes en las diferentes posiciones del campo de juego, dominan muy bien los elementos estratégicos de éste y las acciones tácticas defensivas y ofensivas. El estudio de situaciones tácticas es un proceso integral y sistémico en la vida deportiva; para este estudio se tendrán en cuenta las siguientes etapas: en la etapa inicial con la fase de punto de partida, aplicación de un software de situaciones tácticas con diferentes gradientes de dificultad. Esta primera etapa constituye uno de los componentes del estudio. La segunda fase, denominada de entrenamiento, constituye el momento intermedio para constatación de la relación de los contenidos teóricos-prácticos, con situaciones parecidas a las que se pueden presentar en la competencia. El estudio de situaciones tácticas será sometido al criterio de los especialistas y se aplicó en la selección de fútbol de la Universidad de Pamplona 2015.

Palabras Claves: Fútbol, Situaciones tácticas, Entrenamiento.

\begin{abstract}
In current world football, we find that those teams that are in first place, not only do they have key figures in the different positions on the field of play but they also manage the strategic aspects very well, as well as the tactical, defensive and offensive actions. The study of tactical situations is an integral and systemic process in the life of sports; for this research piece, the following stages are taken into account: - The starting point phase and the phase of implementation, the first constituting one of the components of the study and the second comprising the tactical situations in training conditions. This aspect is established as the intermediate moment that confirms the relation of the theoretical-practical content, with similar situations to those that are present in the competition. The study of tactical situations will be submitted at the discretion of specialists and was applied in Pamplona University's 2015 football team.
\end{abstract}

Keyword: Football, Tactical situations, Training 


\section{INTRODUCCIÓN}

Es muy importante para lograr una excelente trasferencia del entrenamiento a la competición que las situaciones a la ofensiva se entrenen en función de los diferentes posicionamientos en los que el equipo se puede organizar (campo contrario, medio campo, campo propio). Por la importancia que esto tiene para la formación de jugadores que sean capaces de responder a las expectativas y exigencias que impone el fútbol competitivo actual, se hace necesario emprender estudios orientados a conocer los problemas y dificultades del pensamiento táctico de los futbolistas a nivel formativo, con el fin de proporcionarles a los entrenadores mejores métodos $y$ procedimientos para el perfeccionamiento de los conocimientos tácticos y las capacidades resolutivas de los jugadores, todo lo cual puede redundar en la elevación de los niveles de la actividad táctica de los mismos.

Dadas las experiencias obtenidas durante un período de tiempo en la práctica profesional, se pudo detectar, a través de observaciones pedagógicas y de entrevistas realizadas a entrenadores, psicólogos del deporte y directivos, que los futbolistas, a la hora de enfrentar y resolver las situaciones y problemas tácticos que se sucedían durante el juego, presentaban las siguientes insuficiencias: lentitud en el momento de seleccionar una situación lateral 0 de profundidad, toma de decisión incorrecta a la hora de enfrentar una situación táctica imprevista, insuficiente orden táctico a la hora de atacar al adversario, limitaciones para penetrar en la defensa rival con acciones colectivas, demarcación inadecuada en espacios reducidos, e incumplimiento de las tareas orientadas en el sistema táctico planteado.
Estos criterios han sido
preocupación constante de muchos entrenadores nacionales e internacionales de fútbol que preparan atletas en los niveles formativos y de investigadores que se dedican al estudio de los componentes del juego de fútbol entre ellos Roca $B$, \& Josep, A, (1996), Puni Z. A., (1970), Rubinstein, (1978), Williams, (1991), Teplov B M, (2000), Espona, (2008), Colavino, (2002), (González, 1994), Pensado, (2004), Cañizares, (2004), Mariño, (2007), Ortega, (2007) los que han realizado estudios desde diferentes perspectivas; a tal efecto, se han desarrollado algunas investigaciones.

En cuanto a los estudios realizados en Cuba sobre la problemática del pensamiento táctico se encuentran varias investigaciones en el deporte de esgrima; se ha abordado la investigación en dos vertientes fundamentales, una vertiente trata de establecer las influencias de la preparación teórico- táctica sobre los resultados competitivos Williams, (1991). Este estudio quedó reducido al área de laboratorio. La otra trató sobre la efectividad de las acciones del pensamiento táctico en las competencias Bernal, (1994)

Otro estudio realizado acerca de una aproximación al estudio del pensamiento táctico en los esgrimistas fue realizado por Marensa, (2001) y nos revela que el pensamiento táctico en la mayoría de las acciones complejas se caracteriza por un predominio del momento perpetuo sobre el momento selectivo y queda solo en la aplicación y análisis de test psicométricos.

El estudio de Arango, (2000) sobre el análisis del nivel de preparación del pensamiento táctico del béisbol en atletas de la categoría 11 y 12 años del municipio de Holguín nos aporta fundamentalmente que la disminución del tiempo utilizado para las respuestas a los problemas tácticos planteadas, implica un mejoramiento del 
pensamiento operativo de los jugadores y nos ofrece como recomendación que la enseñanza de la táctica debe ser desarrollada en forma de juego.

Un estudio realizado en Argentina por Fuentes, (2001) titulado "Características del pensamiento táctico ofensivo del seleccionado de básquet femenino categoría cadetes de Libertador San Martín", nos manifiesta que el pensamiento táctico de las basquetbolistas estudiadas se caracteriza por la efectividad y eficiencia de las capacidades intelectuales, creativas y resolutivas frente a las diversas situaciones de variado gradiente de dificultad.

Por su parte, Del Pozo (2000), en su estudio de la dinámica ofensiva real del Equipo Nacional de baloncesto femenino cubano en los Juegos Panamericanos de Winnipeg (1999), permite conocer cómo se establece la dinámica relacional del equipo, las combinaciones tácticas y el tiempo de ejecución, datos importantes para la planificación del entrenamiento en cuanto a la preparación de los atletas y el plan táctico. Se plantean consideraciones psicológicas de interés para la preparación táctica de los deportistas de los juegos, con pelotas y de acuerdo con valoraciones del estilo cognitivo del entrenamiento de los problemas de juego, que también resultan útiles para la selección y formación de talentos de base, en razón de las dificultades más comunes con que acceden estos deportistas al Alto Rendimiento.

Vidaurreta, (2002) aporta investigaciones sobre el estilo cognitivo y respuesta táctica, en los deportes con pelota. Expresa que se debe entrenar la detección de las funciones clave definitorias del problema táctico, de manera clara, de forma sistemática, y con retroalimentación conceptual al deportista de modo que le permita crear estrategias personales eficaces para resolver los problemas motores, para facilitar la solución de las dificultades, y preparar el enfrentamiento, y lograr que se expresen sentimientos de satisfacción en relación con el esfuerzo hecho en situaciones de éxito 0 de fracaso, en dicha dirección, a fin de poder establecer los factores 0 indicadores de mayor importancia en la solución de las tareas tácticas individuales, o de grupo, o de equipo y las particularidades psicológicas del pensamiento táctico de los futbolistas. Sin embargo, en la literatura especializada no se han encontrado trabajos de corte metodológico para el estudio de la táctica ofensiva en futbolistas noveles, que aborden un enfoque sistémico y holístico en esta problemática; pues los que existen, se han quedado sólo a nivel de laboratorio 0 , en su defecto, se han centrado específicamente en los aspectos de las acciones tácticas durante el juego.

Sobre la base de los argumentos y criterios anteriormente expuestos se ha llegado a precisar, como problema científico de la presente investigación el siguiente:

¿Cómo estudiar las situaciones tácticas ofensivas en los futbolistas de la Universidad de Pamplona?

\section{MARCO METODOLOGICO}

\section{Metodología}

Para lograr el objetivo propuesto se realizó en primera instancia una revisión universal de los instrumentos existentes para la medición de las situaciones tácticas, se seleccionaron pruebas de laboratorio y de entrenamiento fueron aplicadas a cada uno de los participantes bajo los parámetros del rigor científico. El presente proyecto se realizó con la selección de fútbol de la Universidad de Pamplona 2015. La muestra está constituida por 18 deportistas de sexo masculino. En este sentido, los atletas en cuestión constituyen la cantera directa del deporte de rendimiento de la ciudad. De esta forma, la muestra estudiada presenta un alto nivel de representatividad $y$ confiabilidad de los resultados debido a su alto nivel competitivo. 
Teniendo en cuenta las características de esta investigación, se llevó a cabo en dos fases:

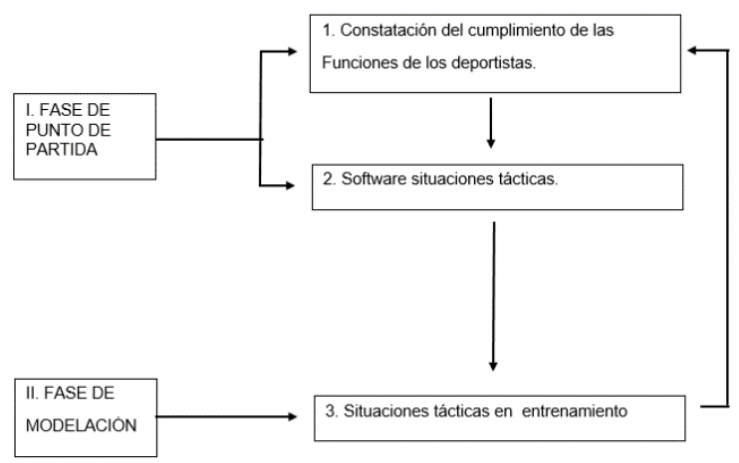

Figura 1. Modelo de Evaluación de Situaciones Tácticas en el Fútbol

Primera fase de punto de partida: Donde se hicieron con la funciones de los deportistas en la ubicación del terreno de juego, lo cual la capacidades resolutivas de los futbolistas constituyen uno de los elementos más importantes para enfrentar las diversas situaciones simples, medias y de mayor complejidad con las cuales interactúa constantemente el deportista y que le permite brindar soluciones a las situaciones tácticas ofensivas durante el entrenamiento y la competencia.

Para la realización del software de situaciones tácticas, el sujeto sentado frente a la computadora se le presentaba una situación de juego en la pantalla, debía analizarla lo más rápido posible y reproducirla en la mayor parte de sus detalles. Se le brindaban diversas alternativas de solución y debía seleccionar creativamente la acción correcta.

Su evaluación era efectuada por el propio software, teniendo como análisis el nivel de la capacidad resolutiva, el porciento, y el tiempo de cada situación.

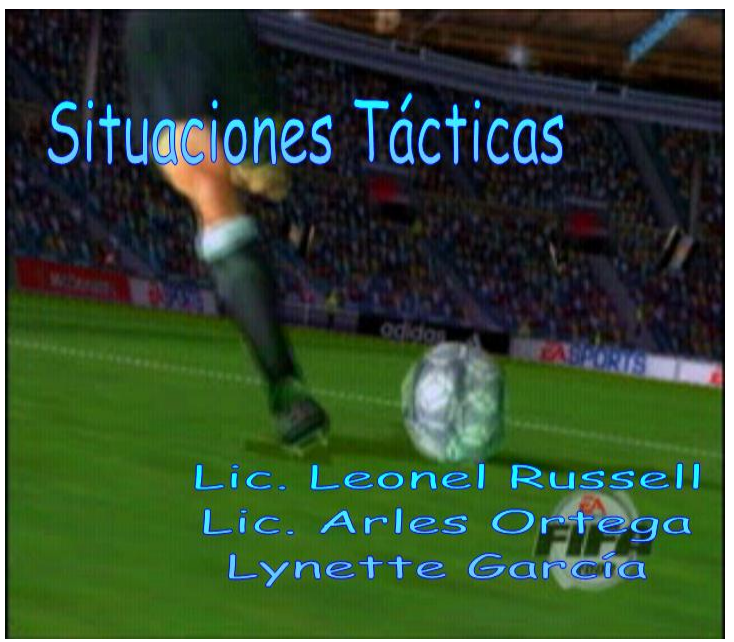

Figura 2. Encuesta Sistemática Situaciones Tácticas

Segunda fase de Modelación: Se realizaron en condiciones similares a las competencias en su disciplina, y se aplicaron diferentes situaciones con gradientes de dificultad. Ejemplo:

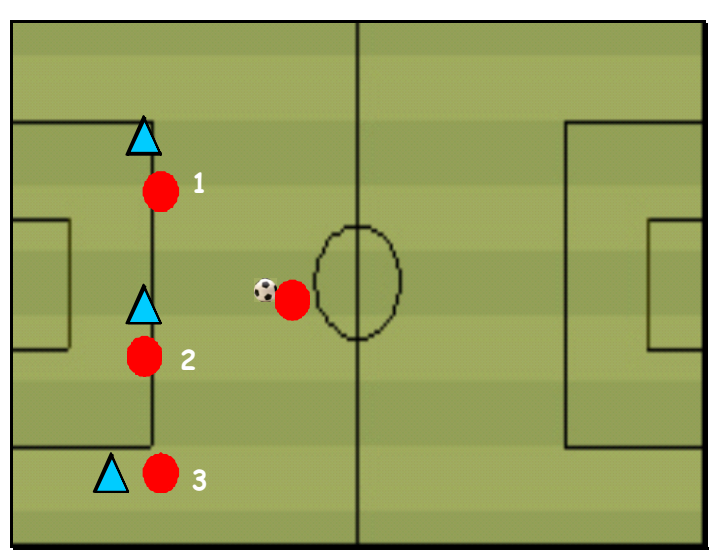

Figura 3. Situación Táctica Ofensiva Simple

Paquete Estadístico: Los datos registrados fueron procesados por el paquete estadístico SPSS 18.0.

Se realizó análisis de tipo descriptivo, correlacionar, pruebas de independencia y análisis de factores con todas las variables, implicadas en la investigación. 
Resultados de la aplicación modelo de evaluación de situaciones tácticas en el fútbol

Es importante señalar que la aplicación del modelo constituye un proceso integral y sistémico que se genera en su etapa inicial con la fase de punto de partida, que abarca la constatación del cumplimiento de las funciones de los futbolistas y la aplicación del software de situaciones tácticas. Esta primera etapa constituye uno de los componentes del sistema del modelo, que con sus resultados debe tributar los elementos potenciales que pueden servir de base para el desarrollo de la capacidad resolutiva que tiene lugar durante la segunda fase de modelación. De esta forma existe una estrecha relación entre ambas fases. De ahí que a cada fase corresponderán diferentes tipos de resultados, pero estos serán parte componente de los subsiguientes. La segunda fase denominada de modelación la cual comprenden las capacidades resolutivas en la solución de las tareas tácticas ofensivas en condición de laboratorio y de entrenamiento, constituye el momento intermedio para constatación de la relación de los contenidos teóricos, con situaciones tácticas parecidas a las que se pueden presentar en la competencia. Es por ello que la aplicación de esta segunda fase resulta de suma importancia pues sirve de preámbulo para predecir la posible manifestación del pensamiento en las condiciones reales. De esta forma los resultados que se derivan son indicadores que deben ser valorados por los entrenadores en interrelación con el psicólogo del deporte.

Tabla 1. Análisis de los resultados en la solución de tareas tácticas ofensivas en condiciones de laboratorio.

\begin{tabular}{|c|c|c|c|c|c|c|c|c|c|}
\hline \multirow{2}{*}{$\begin{array}{c}\text { Software } \\
\text { De } \\
\text { situacione } \\
\text { s tácticas }\end{array}$} & \multicolumn{3}{|c|}{ SISIMPL } & \multicolumn{3}{c|}{ SICOM } & \multicolumn{3}{c|}{ SIMAYCO } \\
\cline { 2 - 10 } & \multicolumn{2}{|c|}{5} & $T^{\prime \prime}$ & $\mathrm{X}$ & $\%$ & $T^{\prime \prime}$ & $\mathrm{X}$ & $\%$ & $T^{\prime \prime}$ \\
\hline Futbolistas & 2 & 40 & 59 & 2.2 & 4,4 & $\begin{array}{c}1,1 \\
2\end{array}$ & 2 & 40 & $\begin{array}{c}1,2 \\
0\end{array}$ \\
\hline $\mathrm{X} \%$ & \multicolumn{2}{|c|}{42} & \multicolumn{3}{c|}{$\mathrm{T}^{\prime \prime} \%$} & \multicolumn{3}{c|}{1,10} \\
\hline
\end{tabular}

$\mathrm{X}$ : Media máxima de efectividad
X\%: Media Grupal

T"\%: tiempo promedio

$\%$ : porcentaje

T": Tiempo en segundos

SISIMPL: Situaciones de complejidad simple

SICOM: Situaciones de complejidad media

SIMAYCO: Situaciones de mayor

complejidad

En la tabla 1 se evalúa la prueba del Software en el laboratorio teniendo como premisa el grado de dificultad creciente de las tareas que conforman la misma. En dicha tabla vemos que los futbolistas en situaciones simples tanto en la cantidad de soluciones correctas brindadas a las diferentes tareas, como en los tiempos empleados en dicha solución, presentan 2 soluciones correctas con un promedio $X=$ $40 \%$ y utilizan un tiempo promedio de 59 segundos. En el caso de las situaciones de complejidad media, a medida que sea el grado de dificultad disminuye el nivel de operatividad. En sentido general los futbolistas investigados presentan 2.2 de soluciones correctas con un promedio $X=44 \%$ a las tareas, con un tiempo promedio de 1.12 segundos. Por su parte en la situación de mayor grado de complejidad los sujetos presentan 2 soluciones dando solución a un menor número de situaciones $X=40 \%$ con un mayor tiempo de 1,20 segundos. En general los deportistas presentan $(42 \%)$ y un tiempo promedio 1.10 segundos.

Tabla 2. Análisis de los resultados en la solución de tareas tácticas ofensivas en condiciones de entrenamiento.

\begin{tabular}{|c|c|c|c|c|c|c|c|c|c|}
\hline \multirow{2}{*}{$\begin{array}{c}\text { Situaciones en } \\
\text { Modelación }\end{array}$} & \multicolumn{3}{|c|}{ SISIMPL } & \multicolumn{3}{c|}{ SICOM } & \multicolumn{3}{c|}{ SIMAYCO } \\
\cline { 2 - 9 } & $\mathrm{X}$ & $\%$ & $\mathrm{~T}^{\prime \prime}$ & $\mathrm{X}$ & $\%$ & $\mathrm{~T}^{\prime \prime}$ & $\mathrm{X}$ & $\%$ & $\mathrm{~T}^{\prime \prime}$ \\
\hline Futbolistas & 3 & 60 & 15 & 3 & 60 & 17 & 3 & 60 & 18 \\
\hline $\mathrm{X} \%$ & \multicolumn{4}{|c|}{60} & \multicolumn{3}{c|}{$\mathrm{T}^{\prime} \%$} & \multicolumn{3}{c|}{17} \\
\hline
\end{tabular}

$\mathrm{X}$ : Media máxima de efectividad

X\%: Media Grupal

T"\%: tiempo promedio

$\%$ : porcentaje

T": Tiempo en segundos 
SISIMPL: Situaciones de complejidad

simple

SICOM: Situaciones de complejidad media

SIMAYCO: Situaciones de mayor

complejidad

La tabla 2 recoge los resultados obtenidos por los futbolistas ante situaciones tácticas modeladas en condiciones reales. En este caso el estudio ha sido diseñado para valorar como se manifiestan las capacidades resolutivas de los deportistas estudiados cuando se encuentran ante situaciones de complejidad simple, media y de mayor complejidad; pero en el propio escenario de entrenamiento y competencia.

En tal sentido observamos que en dicha tabla las situaciones tácticas simples de los futbolistas obtienen valores en cuanto a la cantidad de soluciones correctas brindadas 3 y en cuanto al tiempo empleado para la solución, (15 segundos), una valoración general de estos resultados permite plantear que los futbolistas solucionaron con promedio de $60 \%$ de situaciones tácticas ofensivas.

En el caso de las situaciones de complejidad media vemos que los futbolistas presentan soluciones tácticas en 3 equivalentes a un $60 \%$ así como también ostentan mejores índices temporales empleados en la solución (17 segundos), de forma general, los futbolistas mantienen el nivel de las soluciones correctas con relación a las situaciones simples, aunque no de manera significativa. La valoración de los resultados de los futbolistas en situaciones tácticas de mayor complejidad revela 3 soluciones tácticas correctas con un promedio de $60 \%$, y el tiempo que se ve aumentado en cuanto a la relación de las situaciones.

\section{DISCUSIÓN.}

El estudio realizado por Jaimes, (2012) sobre modelo de evaluación de la efectividad en el baloncesto, se puede evidenciar como principales resultados de la investigación podemos señalar que luego de la aplicación del modelo de los componentes de evaluación de la efectividad $y$ del test en las diferentes etapas, entrenamiento y competencia. Se evidencia que en los resultados obtenidos se lograron significancias en las situaciones reales de juego y las diferencias en los entrenamientos y de las pruebas aplicadas en las sesiones de los mismos, en el cual esta investigación del modelo de evaluación de situaciones tácticas en el fútbol se refleja la elaboración de dos fases relacionadas, la primera fase de punto de partida aplicación de un software de situaciones tácticas con diferentes gradientes de dificultad. Esta primera etapa constituye uno de los componentes del estudio. La segunda fase, denominada de entrenamiento, constituye el momento intermedio para constatación de la relación de los contenidos teóricos-prácticos, con situaciones parecidas a las que se pueden presentar en la competencia.

\section{CONCLUSIONES}

El análisis comparativo de las fases investigadas y de punto de partida, y modeladas del equipo de fútbol, constituyen premisas esenciales en el estudio del pensamiento táctico.

El enfoque utilizado de manera integral en el estudio de las capacidades resolutivas de los futbolistas resulta un procedimiento metodológico e investigativo nuevo, para revelar las premisas del modelo de evaluación de situaciones tácticas en el fútbol.

Las concepciones más actualizadas sobre aspectos metodológicos y experiencias investigativas en el campo de del pensamiento táctico ofensivo en general y de los noveles futbolistas en particular, se debe abordar de una manera integral este proceso en el deporte, por lo que se quedan en el estudio aislado de algunas de las particularidades. 


\section{REFERENCIAS BIBLIOGRÁFICAS.}

Arango, A. (2000). Análisis del nivel de preparación del pensamiento táctico del bésibol en atletas de la categoría 11 y 12 años del municipio de Holguín . La Habana. Cuba: ISCF Manuel Fajardo.

Bernal, H. (1994). Los objetivos de enseñanza en el enrenamiento deportivo. México: Dirección general del deporte selectivo.

Cañizares, H. M. (2004). Psicología y equipo deportivo. Ciudad de la Habana: Editorial Deportes.

Colavino, H. (2002). Estudio de la dinámica relacional de los futbolistas durante las acciones combinadas de ataque . Revista deportes, Argentina, 3:3-4.

Del Pozo, A. (2000). Estudio de la dinámica ofensiva real del Equipo Nacional de baloncesto femenino cubano en los juegos panamericanos de Winnipeg 1999 en el periodo de 1995-2000. La Habana. Cuba: ISCF Manuel Fajardo.

Espona, E. (2008). Fútbol planificación del entrenamiento. Agentina : Edición talleres gráficos Offset Morano.

Fuentes, C. (2001). Características del pensamiento táctico ofensivo del seleccionado de básquet femenino categoría cadetes de Libertador San Martín. Apunts: deportes, 4: 68.

Jaimes , L. (2012). Modelo de evaluación de la efectividad en el baloncesto. ef deportes, 1-4.

Marensa, O. (2001). Aproximación al estudio del pensamiento táctico en los esgrimistas en el periodo 19962001. La Habana. Cuba: ISCF Manuel Fajardo.

Mariño, N. (2007). Análisis del estudio del pensamiento táctico de la selección de fútbol de la Universidad de Pamplona. EFDeportes, 1.

Ortega, A. (2007). Análisis del estudio del pensamiento táctico de la selección de fútbol de la Universidad de Pamplona. EFDeportes, 1.

Pensado, J. (2004). Operatividad táctica en baloncestistas cubanas.

Operatividad táctica en baloncestistas cubanas., (pág. 97). La Habana. Instituto Superior De Cultura Física Manuel Fajardo.

Puni, Z. A. (1970). La preparación Psicológica para las competiciones deportivas. Moscú: Editorial Fiskutura y Deporte.

Roca B, \& Josep, A. (1996). La Teoría en la Psicología a la Educación Física y el Deporte. Barcelona: Editorial Panamericana.

Rubinstein, S. L. (1964). El Desarrollo de la psicología. Principios y métodos. La Habana: Editorial Nacional de Cuba.

Rusell González, L. (1994). Algunas propiedades de los pequeños grupos y colectivos en la educación física y deporte. México: Comisión Nacional de Deporte.

Teplov B M. (2000). El pensamiento del deportista. Barcelona: Editorial Paidotribo.

Vidaurreta, L. (2002). El estilo cognitivo y respuesta táctica en los deportes con pelota . Apunts: Prensa Latina., 1.

Williams. (1991). Psicología aplicada al deporte. Madrid: Biblioteca Nueva. 